\title{
Autoformación: una perspectiva diferente
}

Ángel García del Dujo

José Manuel Muñoz Rodríguez

Universidad de Salamanca

\footnotetext{
Correspondência:

Ángel García del Dujo

Universidad de Salamanca

Facultad de Educación

Paseo de Canalejas, 169

37008 - Salamanca - España

e-mail: agd@usal.es
}

\section{Resumen}

El objetivo de este trabajo es el de mostrar que una de las competencias que hoy día deben fomentarse, si queremos responder realmente a las demandas laborales desde una perspectiva socioeducativa, es la de la autoformación. Nadie pone en duda que el modelo de desarrollo que se pretende implementar en la sociedad actual requiere una mayor masa formativa, un mayor nivel de formación en cada individuo. No obstante, pensamos que también requiere un modelo de formación, sino diferente sí, al menos, complementario. Más allá de una pedagogía individualista, mentalista, aislacionista y abstracta, basada en una lógica de conocimientos e instituciones, pensamos que el sistema formativo debe recuperar una visión más social y global de los procesos de producción y de la vida. Una lógica que esté apoyada en los modos de acción que tienen lugar en el mundo de la vida y del trabajo. Sobreponiéndonos a los simples listados de competencias, nos hemos preguntado por la forma como se generan, adquieren y desarrollan las competencias, concluyendo que el enfoque de la autoformación responde no sólo a determinadas tendencias económicas sino a las transformaciones que viene conociendo la sociedad en la última década. Teniendo en cuenta que el ser humano es algo más que un nódulo productivo, laboral, y la autoformación es una característica que se predica del sujeto en cuanto tal, en su totalidad y en relación con su entorno, concluimos el trabajo presentando la autoformación como competencia clave y como modelo de formación para la sociedad del siglo XXI.

\section{Palabras-clave}

Competencias clave - Autoformación - Mercado laboral - Sistema formativo. 


\section{Self-formation: a different perspective}

Ángel García del Dujo

José Manuel Muñoz Rodríguez

Universidad de Salamanca
Contact:

Ángel García del Dujo

Universidad de Salamanca

Facultad de Educación

Paseo de Canalejas, 169

37008 - Salamanca - España

e-mail: agd@usal.es

\section{Abstract}

The objective of this work is to show that one of the competences that nowadays must be fostered, if we really want to respond to the demands for workforce from a socioeducational perspective, is that of self-formation. There is little doubt that the development model that society currently wants to implement requires a larger formative volume, a higher level of formation of each individual. However, we also think that it demands a model of formation which, if not different, is at least complementary to that one. Looking beyond an individualist, intellectualist, isolationist, and abstract pedagogy based on a logic of knowledges and institutions, we believe that the formative system must rescue a more social and global view of the processes of production and of life. A logic grounded on the modes of action that take place in the world of life and work. Overcoming the simple lists of competences, we have asked ourselves how the competences are generated, acquired and developed, and we have concluded that the approach of selfformation responds not only to certain economic trends, but also to the transformations that society has experienced along the last decade. Keeping in mind that the human being is more than just a productive, labor node, and that self-formation is a characteristic expected of the subject as such, in his/her totality and relation with the surroundings, we conclude this work by presenting selfformation as a key competence and model of formation for the society of the $21^{\text {st }}$ century.

\section{Keywords}

Key competences - Self-formation - Work market - Formative system. 


\section{Planteamiento de la cuestión}

Tanto el debate como la investigación sobre la relación que se establece entre formación y trabajo tienen ya un largo recorrido. Desde la época en que la educación de una persona comienza a ser considerada como un bien de utilidad pública, por entenderla como instrumento para el desarrollo económico de los pueblos, todas las sociedades han sacado a la superficie, periódicamente, el problema de la adaptación entre el sistema educativo, sobre todo en sus tramos de profesionalización, y el mundo laboral (Comisión Europea, 2002).

La tensión entre estos dos polos ha sido constante a lo largo de la historia, si bien es cierto que ha habido momentos en los que esa tensión ha conocido un notable incremento por razones procedentes normalmente del mundo laboral. Y la conclusión es bastante clara y recurrente: aunque se admite que los sistemas formativos configuran buena parte del sistema productivo, la realidad es bien distinta, pues no está demostrado, de forma concluyente, la correlación que debiera existir.

Situados en la sociedad de la información y del conocimiento, esos momentos de alta tensión se suceden con más frecuencia, precisamente porque las transformaciones de los procesos productivos se han acelerado como consecuencia de la velocidad con la que tienen lugar los cambios en las tecnologías de los procesos de producción. Más aún, en nuestros días, como los períodos de transformación de estas tecnologías son muy cortos, tenemos la sensación de que vivimos en un estado de cambio permanente.

Una situación, a la par que un problema, que, si bien viene de lejos, presenta hoy nuevas particularidades que son, en definitiva, las que configuran y describen los retos que tiene ante sí el sistema formativo: cambios demográficos y de estructura poblacional - incorporación de la mujer al mundo laboral, baja natalidad, nueva ola de inmigración; cambios económicos, más globales y en reciclaje continuo - desplazamiento del eje productivo desde el sector indus- trial al sector servicios; cambios tecnológicos y comunicacionales y cambios, incluso, en la propia estructura de los ciclos vitales, pues el tradicional modo de entenderlos - formación/ trabajo/jubilación - está en crisis.

Entre tanto, las personas continúan aferradas a un sistema educativo sin tener muy claro hacia dónde se encaminan y si han tomado el camino correcto, ya que el sistema sigue sin mirar de frente al mercado laboral y sin encontrar definidos los ejes de conexión. Y esto es así, entre otras razones, porque nos hemos aferrado a un patrón cuya columna vertebral se basa en un modelo de heteroformación que guía y vigila todo el proceso formativo de una persona. Desde la fase de diagnóstico de las necesidades de formación hasta el tratamiento de esas necesidades, pasando por los lugares, tiempos y modos de acción formativa, el itinerario formativo, en la mayoría de los casos, viene establecido, adoptando un sistema de formación poco realista y escasamente adaptado a las demandas de los empleadores y a las necesidades formativas de los sujetos.

Nadie pone en duda que el modelo de desarrollo que se pretende implementar en la sociedad actual requiere una mayor masa formativa, un mayor nivel de formación en cada individuo. No obstante, pensamos que también requiere un modelo de formación, si no diferente, al menos complementario. Más allá de una pedagogía individualista, mentalista, aislacionista y abstracta, basada en una lógica de conocimientos e instituciones, entendemos que el sistema formativo debe recuperar una visión más social y global de los procesos de producción y de la vida. Una lógica que esté apoyada en los modos de acción que tienen lugar en el mundo de la vida y del trabajo.

Con este planteamiento de fondo, la idea que buscamos presentar es la siguiente: una de las competencias que hoy deben fomentarse, si queremos responder realmente a las demandas laborales y retomar la perspectiva social señalada, es la de la autoformación. Se trata de una mutación pedagógica que pone más énfasis en la 
capacidad de actualización, adaptación y uso de conocimientos, procedimientos y comportamientos, que en la mera adquisición de los mismos, acorde con un status diferente del individuo en cuanto ciudadano y productor congruente con los cambios económicos, tecnológicos y sociales de nuestro tiempo.

La perspectiva predominante en el tratamiento de la autoformación ha sido y es, ciertamente, laboral, económica, como puede comprobarse en la numerosísima documentación que el tema de la formación continua y sus análogos ha generado en los últimos treinta años, también en buena parte de la no menos numerosa documentación evacuada por las distintas instancias de la Comunidad Europea; el principio y los fundamentos de la autoformación aparecen hoy estrechamente relacionados con el mundo del trabajo (Carré; Moisan; Poisson, 1997; Martin; Savary, 1998; Hammer; Cahmpy, 1994; Comisión Europea, 1999a; 1999b; 1999c, 2003).

Pues bien, nuestro objetivo en este trabajo es abrir una vía de análisis diferente: presentar la autoformación como perspectiva global del proceso de desarrollo del ser humano, incluso como proceso general de desarrollo de la especie humana. El ser humano es algo más que un nódulo productivo, laboral, y la autoformación es una característica que se predica del sujeto en cuanto tal, en su totalidad y en relación con su entorno, de un individuo en cuanto miembro de un grupo y del grupo como tal.

Aunque la evolución del conocimiento y la historia de las formas y procesos de producción hayan desembocado en este constructo y hayan enfocado su análisis bajo el principio de la rentabilidad económica, queremos presentar la autoformación en cuanto competencia del sujeto y grupo humano, una propiedad del individuo como persona, trabajador y ciudadano, una característica requerida hoy casi más por razones sociales que económicas. En definitiva, una exigencia y posibilidad de la sociedad de nuestro tiempo antes que una metodología didáctica coherente con una estrategia de supervivencia y desarrollo económico.

\section{Mercado de trabajo y sociedad}

Aunque buscamos una perspectiva más amplia para el tratamiento de la autoformación, no conviene ignorar la dinámica laboral característica de nuestra sociedad. $Y$ aquí son muchos los temas y problemas que hacen acto de presencia: altas tasas de paro, demandas laborales, desequilibrios económicos, desigualdades territoriales, planes formativos, acuerdos sectoriales etc. Si, además, todos esos temas adquieren uno $u$ otro perfil en función de la perspectiva de análisis, hemos de admitir que estamos ante una temática compleja, dinámica, muy amplia; sin ninguna pretensión de exhaustividad señalaremos algunas características que definen el estado actual del mercado de trabajo para advertir cómo influyen y son influenciadas por la sociedad.

Un primer aspecto que hay que resaltar viene dado por la globalización de la economía e internacionalización de los mercados, cuya consecuencia principal termina siendo la interdependencia entre las grandes empresas y entre los propios mercados de trabajo (Fujii; Ruesga, 2004). La creación del mercado único europeo, como exponente principal de lo que es una economía entendida desde una perspectiva internacional, marca un devenir en los itinerarios laborales amplio y exigente; se amplía el mercado laboral pero a la vez se complica, pues aparecen unas competencias nuevas y desaparecen otras.

En segundo lugar, reconocer que la calidad del empleo se ha ido alterando de forma sustancial. En estos momentos es difícil, en un alto porcentaje, hablar de empleo único y fijo como uno de los elementos exponenciales del mercado de trabajo. Por el contrario, la tónica dominante es el empleo inestable, cambiante, diverso y parcial; se llega a prolongar el período de búsqueda de empleo, aun estando incorporado al mercado de trabajo. Hechos todos ellos que conllevan una fuerte inestabilidad laboral y personal a la que, de momento, no estamos acostumbrados. 
Esta alteración tiene como elemento más característico, a la vez que preocupante, la existencia de paro. No podemos quedar impasibles sólo por el hecho de que casi siempre ha existido, que casi nadie ha conseguido extinguirlo y que la mentalidad de la sociedad está habituada a ello. La realidad está ahí; las cifras son claras y algunos sectores, jóvenes principalmente, y no sólo los que presentan un bajo nivel de formación, soportan una alta tasa de paro (Albert et al., 2003). Concretamente, entre nosotros encontramos que:

Por tramos de edad, son los que tienen entre 25 y 29 años, los jóvenes que en mayor medida se encuentran en situación de desempleo, tanto los hombres como las mujeres, mientras que en el tramo de 30 a 34 años, hay menor número de desempleados. Entre los parados que buscan su primer empleo destacan los jóvenes con edades comprendidas entre 20 y 24 años. $Y$ el tiempo medio que tarda un joven en encontrar un puesto de trabajo es, principalmente, superior a los 6 meses e inferior a un año, aunque hay un porcentaje de jóvenes que tarda más de dos años en encontrar un empleo. (U.G.T., 2004, p. 5)

Así mismo, los sectores laborales más demandados han sufrido un giro considerable. En términos generales, la industria y la manufactura han perdido el protagonismo que han tenido en estos últimos años dando paso al sector servicios como ámbito en el que con mayor facilidad se encuentra empleo. El comercio y la hostelería junto a la construcción, son algunos de los sectores cuyo crecimiento del empleo es superior a la media.

En este campo existe un amplio reconocimiento del proceso de globalización económica, proceso que se acompaña de la industria hacia los servicios. Esta terciarización de la sociedad implica un aumento del paro vinculado a la pérdida de peso de ciertos sectores productivos, que conlleva la aparición de nuevos fenómenos de exclusión, y un reconocimiento de la falta de adecuación de la formación actual. (Petit; Badosa; Mampel, 2002, p. 201)

Junto a estas tendencias, mencionaremos también la aparición de nuevos yacimientos de empleo que, de unos años a esta parte, están adquiriendo protagonismo. De un lado, hemos de anotar la aparición del llamado sector cuaternario cuya principal consecuencia es el denominado teletrabajo. Una forma de modernizar la organización del trabajo, haciéndolo más flexible, con el objetivo de mejorar la productividad y la competitividad en base a un equilibrio entre flexibilidad y seguridad (lzquierdo, 2006).

El teletrabajo es una forma de organización y/o realización del trabajo, utilizando las tecnologías de la información en el marco de un contrato o de una relación de trabajo, en la cual un trabajo que podría ser realizado igualmente en los locales de la empresa se efectúa fuera de estos de forma regular. (Acuerdo Marco Europeo sobre Teletrabajo firmado el 16 de Julio de 2002 en Bruselas)

Y, de otro lado, llevamos un tiempo en que van apareciendo nuevos sectores de empleo; nos estamos refiriendo a aquellas profesiones que tienen que ver con el medio ambiente, la seguridad, el consumo, la higiene o, incluso, desde hace más tiempo, las relacionadas con los servicios sociales. Más aún, el amplio abanico de posibilidades que comienzan a sostenerse en el mercado de trabajo incita a desarrollar un fenómeno, que ha existido desde siempre en cuanto formas de trabajo independiente, pero que empieza a tomar un cariz distinto en los tiempos actuales: el autoempleo, como opción idónea para profesionales emprendedores de cara a abandonar el desempleo o como filosofía de promoción personal (Alles, 2006).

Un cuarto aspecto que no podemos dejar de mencionar atañe a los colectivos poblacionales. En este sentido, el mercado de trabajo actual viene definido por diferentes movimientos. Por una parte, nos encontramos con nuevos 
núcleos poblacionales, de convivencia, que se perciben igualmente tanto en las plazas públicas como en el ámbito laboral. Los continuos procesos migratorios con los que convive la sociedad actual invitan, cuando menos, a analizar el mercado de trabajo desde esta perspectiva poblacional novedosa pues, en cierta medida, influye y reorienta algunos sectores laborales'.

Y junto a la diversidad hemos de mencionar también los cambios en la estructura de los ciclos vitales. Como ya hemos indicado, el tradicional proceso de formación-trabajo-jubilación está en crisis (Moreno, 2003). Más aún, parece existir acuerdo en la poca flexibilidad que se viene percibiendo por parte del sistema formativo para adaptarse a estos ritmos y necesidades distintas de las tradicionales, sobre todo si tenemos en cuenta que la estructura demográfica actual viene definida por un incremento de la tercera edad y una menor tasa de natalidad.

Y, en último término, si bien es una cuestión a la que no se le está dando toda la importancia que tiene, se percibe, principalmente a edades juveniles, un desconocimiento de la realidad laboral. Las personas desconocen no sólo los cauces a través de los cuales se puede optar por el itinerario más óptimo para insertarse en el mercado de trabajo sino, más aún, se ignora el propio mercado de trabajo. En parte porque no existen mecanismos de orientación acordes con la realidad laboral por parte del sistema formativo y en otra parte porque el sistema de formación seguido, heteroformación, facilita dicho desconocimiento.

En suma, una serie de características, movimientos y tendencias económicas y sociales, culturales, del mercado de trabajo que están demandando un mejor engranaje entre el sistema formativo y el sistema social. No son todas las que pudieran estar, pero sí las más emblemáticas en estos momentos, cuya tónica dominante viene a resaltar que vivimos en un tiempo globalizador, postindustrial, neocapitalista (Sarramona, 2002), que implica una serie de cambios acelerados respecto a lo que fue el final del siglo XX. Otros tiempos, otros problemas que requieren solucio- nes distintas, novedosas, entre las que se encuentra esa otra perspectiva que estamos reclamando para la autoformación que detallaremos más adelante.

\section{Mercado de trabajo y sistema formativo: treinta años de investigación}

Junto a la sucinta descripción del mercado laboral que acabamos de hacer, estamos obligados también a preguntarnos hacia dónde nos ha llevado la investigación en este último cuarto de siglo para, por un lado, comprobar, como ya apuntábamos en la introducción, que venimos estando excesivamente apoyados en un enfoque economicista sobre educación y empleo, y, por otro, para dar un paso adelante en el estado que presenta actualmente esa investigación. Este recorrido será el que nos permita aportar un cambio de orientación coherente con las exigencias de nuestra sociedad y acorde con las teorias socioeducativas de nuestro tiempo, que nos facilite presentar, posteriormente, la autoformación desde una concepción más amplia.

\section{De la fase de cualificación profesional...}

El maridaje llevado a cabo por el sistema formativo y el mercado laboral a lo largo de la historia ha venido definido por un proceso fácil de ver e incluso de soportar: todo cambio en la tecnología y en los procesos de producción y conocimiento han venido acompañados de mo-

1. Esta tendencia lleva consigo el denominado "mercado sin papeles" o trabajo marginal, cuyo incremento empieza a tomar medidas incontrolables. Un tránsito de trabajadores necesitados, cuyo objetivo es la subsistencia, que conlleva, aunque no necesariamente pero sí con frecuencia, la llamada economía sumergida. Una situación que todo gobierno intenta normalizar poniendo una serie de condiciones cuyos resultados son más bien despidos por parte de los empleadores que contratos en regla a favor de los inmigrantes. Pero algunos avances se empiezan a hacer a este respecto. En el estado español, el lunes 7 de Febrero de 2005 comenzaba un proceso de normalización establecido en la Disposición Transitoria Tercera del nuevo Reglamento de la Ley orgánica 4/ 2000, de 11 de Enero, sobre derechos y libertades de los extranjeros en España y su integración social, plazo que se extendió hasta el 7 de Mayo de 2005 y en el que se presentaron 690.679 solicitudes. Disponible en: <http:// www.mtas.es/balance/>. Consultado en: 28-04-2006. 
mentos de relevo generacional que, a su vez, aprovechaba el sistema educativo para introducir transformaciones metodológicas y conceptuales. Los cambios experimentados por el trabajador no eran traumáticos, al producirse más bien en los inicios de su trayectoria laboral, además de que no toda la masa laboral quedaba afectada por las innovaciones producidas (Rodríguez, 2006).

En las últimas décadas, el proceso ha sufrido un giro importante, de manera que el trabajador queda sometido a posibles transformaciones y reorientaciones en cualquier momento de su trayectoria laboral y, además, como indicábamos con anterioridad, con muchas probabilidades de que esta situación ocurra varias veces en su vida laboral. El argumento se repite una y otra vez: la inevitable rapidez con la que se producen los cambios en las tecnologías de producción, lo que implica un proceso de adaptación e incertidumbre permanente.

Buena parte, pues, de responsabilidad de esta dinámica se hace recaer, como estamos viendo, sobre la variable tiempo. Son los cada vez más cortos períodos de tiempo de estabilidad de la tecnología y estructura productiva los que actúan de motor de esta dinámica. Una vez más en la sociedad postmoderna se acude al tiempo y a la velocidad con la que se suceden las fases como factor explicativo; la concentración de fenómenos en breves períodos de tiempo tendría un efecto multiplicador de los cambios con capacidad de generar transformaciones cualitativas.

Éste ha sido el esquema predominante de reflexión hasta hace poco y, en función de esta perspectiva de análisis, la investigación ha buscado reiteradamente la forma de adaptar lo mejor posible las características de la formación a la demanda laboral. Las versiones o derroteros que ha seguido esta línea de investigación han sido varias, pero siempre en la misma dirección: con diferentes perspectivas, metodologías y técnicas, se ha estudiado durante más de veinte años la oferta y demanda de educación superior y su adecuación a las características socioeconómicas de la realidad en la que se insertaba, así como los procesos de inserción laboral. El objetivo era ajustar cuantitativa y cualitativamente la demanda de plazas en la enseñanza postobligatoria, en función de las motivaciones e intereses profesionales y vocacionales de los alumnos y la oferta del mercado laboral ${ }^{2}$.

Esta línea de investigación no ha estado exenta de valoraciones críticas, como consecuencia, a su vez, de las sucesivas reconsideraciones a que se ha visto sometida desde la década de los setenta la teoría del capital humano, que en el fondo la sustenta (López y Grandio, 2005; Johones, 1995). Se ha cuestionado la interpretación de una educación excesivamente adaptativa y utilitarista que lleva consigo, se ha dudado de la existencia y correcto funcionamiento de la necesaria bidireccionalidad en los flujos de información entre el mundo formativo y el mundo laboral, se ha reconocido que este enfoque implica necesariamente un desfase temporal en el comportamiento del sistema educativo respecto de las exigencias del mundo laboral... pero durante veinte años ha sido la línea dominante en este campo.

La década de los ochenta y parte de los noventa estuvo repleta de altas tasas de desempleo, despidos en bloque, reciclajes formativos forzosos... Indicadores todos ellos de una estructura y dinámica económica sacudida por fuertes reconversiones y reestructuraciones empresariales, pero no fueron suficientes para poner en entredicho un modelo de investigación lineal, cuantitativo, de las relaciones formacióntrabajo. Todavía no se cuestionaba el modelo productivo en su totalidad y no se percibía que estábamos iniciando una nueva era en los modos y formas de producción, gestión y distribución de bienes y servicios, al igual que tampoco se

2. En algunos países, como en España, esta línea de investigación se ha visto reforzada por un contexto de nueva configuración política y administrativa, con la pretensión consiguiente de diseñar un sistema educativo que respondiera a las necesidades socioeconómicas de su entorno más próximo. Ley Orgánica 5/2002, de 19 de Junio, de las Cualificaciones y de la Formación Profesional. (BOE de 20 de Junio de 2002). R. D. 1128/2003 de 5 de Septiembre por el que se regula el Catálogo Nacional de Cualificaciones (BOE de 17 de septiembre de 2003). A este respecto recomendamos la lectura de Casal, Colomé y Comas, (2003). 
cuestionaban los conocimientos que se entendían necesarios y los modos de producción y adquisición de esos conocimientos.

En estos tiempos la investigación concluyó en la necesidad de una mayor cantidad de formación, de una mayor masa formativa para acometer las transformaciones económicas, la necesidad de una formación continua acorde con los requisitos derivados de esas transformaciones $\mathrm{y}$, si acaso, la necesidad de una formación diferente cuya explicitación nadie se atrevía a concretar y que se diluía en controversias que en ocasiones rozaban el bizantinismo (Lope; Martín, 1995). No se cuestionaba la forma escolar de tratar las exigencias socioeducativas de la economía, ya se llevase a cabo dentro o fuera del sistema educativo, dentro o fuera del sistema empresarial.

Y del sujeto, en cuanto futuro o actual trabajador, se pedía la mayor disponibilidad, movilidad, formación y colaboración para el cambio, tanto para el cambio de trabajo como para someterse a los indispensables procesos de reciclaje que normalmente se le proporcionaban ya empaquetados y que se iban a desarrollar bajo modelos individualistas o grupales, dentro o fuera del sistema educativo, pero siempre didactistas y escolares.

En definitiva, lo que trasluce a partir de las interrelaciones establecidas entre el sistema formativo, el mundo laboral y las personas implicadas es un modelo de heteroformación que guía y vigila todo el proceso formativo de un sujeto, desde la fase de diagnóstico de las necesidades de formación hasta el tratamiento de esas necesidades, pasando por los lugares, tiempos y modos de acción formativa. Expresado en otros términos, que al sujeto, ya se encuentre en estado puro de formación, de desempleo o de trabajo, se le indica y proporciona cuánto y qué tipo de formación necesita así como también se le señala el lugar o lugares y procedimientos para adquirirla. Mientras tanto, los contenidos formativos siguenn siendo los mismos, si acaso con un punto más de cercanía y proximidad temática a las características de los puestos de trabajo. Eran los tiempos en que el panorama laboral y formativo estaba presidido por el concepto de cualificación profesional.

\section{... a la fase de las competencias}

Decíamos al principio que las relaciones entre el sistema educativo y el sistema productivo nunca fueron fáciles; en esta segunda era moderna, como prefieren muchos llamarla, tampoco lo son o quizás lo sean menos por la rapidez y persistencia de los cambios a los que estamos sometidos. En este sentido cabe pensar que lo que genéricamente se conoce con el nombre de problema educativo siempre fue el mismo y en términos muy sencillos podríamos concretarle en las relaciones que el sistema mantiene con su entorno. De la mayor o menor amplitud de ese entorno y su tipología así como de la cantidad y diversidad de dimensiones que queden incluidas en esa relación dependerá, a su vez, la mayor o menor complejidad y problematicidad de dicha relación (García; Martín; Pérez, 2000) ${ }^{3}$.

En los últimos años, una serie de cambios en los procesos productivos y tecnológicos y, sobre todo, en el entorno, han terminado por cuestionar el enfoque tradicional de la relación entre educación y producción, entre trabajo, empleo y formación, un enfoque que se apoyaba básicamente en el concepto y en el principio, aplicado estática y mecánicamente, de cualificación técnica del trabajador y de actualización permanente de esa cualificación o reciclaje periódico. Lo cual no significa que esa cualificación no se considere hoy ya necesaria, pero sí que se empieza a considerar insuficiente.

Más aún, la investigación comienza a dejar entrever que el calificativo de 'técnico' asignado a esas cualificaciones $y$, en general, a

3. Este documento pertenece a la investigación "Key Competences in Vocational Education and Training. Towards the 21 st Century. Promoting mobility" aprobada y financiada por la COMISION DE LAS COMUNIDADES EUROPEAS. DIRECCION GENERAL XXII. EDUCACION, FORMACION Y JUVENTUD. Programa Leonardo da Vinci. Contrato: P/97/1/35005/PI/ I.1.1.a/FPI, desarrollada por investigadores y profesionales de diversos organismos, centros e instituciones educativas, empresariales y sindicales de Portugal, España, Dinamarca y Países Bajos entre 1997 y 2001. 
la formación de los trabajadores puede resultar inadecuado cuando nos adentramos en el campo de las nuevas tecnologías, por ser una categoría estrechamente conectada a tecnologías antiguas basadas en la ingeniería mecánica y eléctrica (Wilson, 2001).

En cualquier caso, ya sea por insuficiencia o por inadecuación de lo que en su momento se entendió como cualificación, lo cierto es que ya desde hace unos años se viene manejando, aunque todavía más en la literatura que en la realidad, un nuevo concepto -competencia- que pretende paliar los déficits de aquel viejo término por la vía de introducir una mayor diversificación no sólo en la tipología sino también y sobre todo en la naturaleza y orientación de las cualificaciones (Tagliagambe, 2002).

El desfase permanente entre educación y trabajo que de manera inevitable genera el modelo anterior de detección y tratamiento de las necesidades formativas, incrementado hoy por el ritmo en que se producen los cambios tecnológicos y la facilidad para aplicar a todos los componentes del proceso productivo esas tecnologías, de forma que la desincronización entre formación y trabajo se hace visible ya en los mismos inicios de la vida laboral de un trabajador, invita a pensar en un nuevo modelo de diagnóstico de las exigencias formativas. Un nuevo tipo de rasgos, características, calificaciones, del trabajador más acordes con la nueva naturaleza de los procesos de producción $\mathrm{y}$, sobre todo, formas diferentes de adquisición y fomento de esas características. Problemas o retos de nuestro tiempo que lentamente están conduciendo a un nuevo modelo de formación.

Lo que estamos queriendo plantear es que más allá de las acusaciones referidas a una oferta formativa demasiado estrecha, teórica y especializada e incluso insuficiente, por no contemplar otros aspectos que se entienden de interés en el mundo del trabajo y ante las que el sistema educativo responde ampliando la oferta, lo que se está solicitando del sistema educativo es que lleve a cabo cambios de enfoque y orientación. Y para ello hay que empezar por el reconocimiento de que en nuestros días hay más contextos de aprendizaje y formación que el propio ámbito escolar, que la empresa y la sociedad de hoy y del futuro se han convertido en instancias generadoras y renovadoras de conocimientos y que las situaciones de trabajo y de aprendizaje tienden a confluir desde el momento en que se han hecho presentes las nuevas tecnologías de la información y la comunicación.

Dicho en otros términos, lo que se está pidiendo de la educación, y muy concretamente del sistema educativo, es que abandone una Pedagogía individualista y mentalista, basada en la lógica de los conocimientos y de las instituciones, y que recupere una visión más global y social de los procesos de producción y de la vida, una lógica apoyada en los modos de acción que tienen lugar en el mundo de la vida y del trabajo.

Y buena parte de este cambio de rumbo puede venir de la mano del concepto de competencia, porque está implícito en ese concepto (Fernández, 2006). No se trata simplemente de un concepto ambiguo, uno más del convoy semántico que forman otros términos como polivalencia, empleabilidad... y que ha aparecido en el campo educativo ante el desconcierto generado por las exigencias del mercado laboral; no estamos ante una simple relectura revisada y actualizada de enfoques tayloristas y fondistas, combinada con otros de relaciones humanas. Se trata, más bien, de una mutación pedagógica que pone mayor énfasis en la capacidad de actualización, adaptación y uso de conocimientos, procedimientos y comportamientos, que en la mera adquisición de los mismos, acorde con un status diferente del individuo en cuanto ciudadano y productor congruente con los cambios económicos, tecnológicos y sociales de nuestro tiempo.

\section{Modos de generación y adquisición de competencias: más allá del inventario}

Comienzan a abundar ya las investigaciones que presentan un inventario amplio, muy amplio, 
de competencias requeridas, ya sea a nivel de formación primaria, secundaria o superior postobligatoria, donde además el elenco de competencias enumeradas es tan diverso como amplio (Sarramona, 2000; 2004; Alles, 2005). Escasea, en cambio, la reflexión crítica sobre los modelos utilizados para sacar a la superficie unas u otras competencias y, sobre todo, poco se dice de la forma de certificar la existencia de unas u otras y mucho menos de la forma como se adquieren o no, se enseñan o no, dichas competencias, cuestiones estas que interesa mucho considerar para no caer en el modelo que tratamos de evitar y que hemos descrito anteriormente. Porque, también por la vía de las competencias, podemos estar moviéndonos en un enfoque un poco más concreto pero tan escolar y heterónomo como el anterior.

No negamos, por otra parte, que en el fondo del enfoque de las competencias se encuentre un aprovechamiento económico por parte de la empresa de los recientes avances de las ciencias sociales y las ciencias cognitivas, utilización que, por otra parte, entendemos legítima, pero pensamos que una visión global de este planteamiento no puede quedar reducida a esos límites. Apreciamos en este sentido un conjunto de rasgos diferenciadores que no sólo obedecen a contextos económicos, tecnológicos y sociales diferentes sino que llevan consigo también una visión y una interpretación distinta de lo que debe ser la educación y la Pedagogía en este siglo XXI.

\section{Un enfoque interrelacional como matriz generadora de competencias $^{4}$}

Estamos defendiendo que esta línea de reflexión no es una simple vuelta a las perspectivas clásicas de la importancia del capital humano, dadas las transformaciones cuantitativas y cualitativas que vienen produciéndose en el ámbito laboral y que han terminado por afectar al propio concepto de trabajo y, por supuesto, al de formación. Son esas transformaciones precisamente las que constituyen el contexto de introducción de un nuevo concepto para referirnos al tipo de formación requerido de los trabajadores.

En efecto, nadie pone en duda que el modelo de desarrollo que se pretende a finales de siglo requiere una mayor masa formativa, un mayor nivel de formación en cada individuo, pero sobre todo un tipo de formación diferente; esta afirmación, que antaño terminó reconduciéndose hacia el modelo predominante, como hemos señalado anteriormente, resulta hoy incuestionable, aun reconociendo su enorme complejidad, pues no resulta nada fácil delimitar y concretar ese nuevo y diferente tipo de formación requerida.

Durante mucho tiempo, cuando se trataba de caracterizar el tipo y modo de formación aludidos con esa expresión, aparecían enormes dificultades para avanzar un poco más allá de lo que era la mera repetición de principios: "el usufructo del potencial humano en toda su extensión, - el conjunto de los ciudadanos -, y en toda su intensidad - capacidades individuales - equivale a una comprensión de la existencia y del sujeto con arreglo a nuevas dimensiones" (Consejo Económico y Social de las Comunidades Europeas, 1996). Era un tanto ingenuo aceptar sin más esas formulaciones de principios, pues interesaba conocer cuáles eran esas capacidades y las nuevas dimensiones en las que quedaba situado el ser humano, pero no resultaba fácil encontrar la estructura de pensamiento que nos condujese a ese mapa de capacidades y dimensiones diferentes.

El primero en experimentar dificultades para definir la tipología de esa formación era el propio mercado de trabajo. No es la primera vez que el sistema productivo reconoce que resulta complicado para las empresas comprender el tipo de cualificaciones que han de fomentarse. Por tanto, es difícil establecer un análisis concreto y a medio plazo sobre las necesidades de formación y las previsiones de

4. Pensamos que es tanto o más importante la reflexión sobre el enfoque que los ya numerosos listados de competencias que empiezan a proliferar porque sólo así descubriremos si estamos ante una perspectiva diferente no ya de las relaciones entre formación y trabajo, sino también, y sobre todo, de la forma de concebir hoy la educación y la sociedad. 
las condiciones de mano de obra (Comisión Europea, 1993). Fueron precisamente esas dificultades las que llevaron al mercado a insistir en conceptos tan amplios como formación de base, versátil, flexible, polivalente, adaptativa..., cuya importancia no radicaba tanto en la concreción que hacian, más bien escasa, del tipo de formación requerida para el trabajo, cuanto en la alusión a un cambio de mentalidad en el comportamiento y formación global del individuo, que quedaba afectado así en todas sus dimensiones y capacidades. Eran los momentos de balbuceo de un modo distinto de afrontar la situación (Homs; Resines, 1999; Lázaro; Martínez, 1999).

Así, en cualquier estudio sobre la estructura y funcionamiento de la realidad económica de nuestro tiempo, por muy somero que fuese, empezaban a apuntar una serie de rasgos relativos a los modos de organización, a las formas y procesos de producción y, sobre todo, a las herramientas de producción que constituian otros tantos referentes para definir ese nuevo tipo de formación exigida.

No estábamos ya ante meros cambios estructurales y organizativos de las empresas, ni siquiera ante la aparición de nuevos sectores productivos, que también incidieron en una diferente actuación del elemento humano en el proceso laboral; se trataba más bien de la ubicación del trabajo y del complejo 'tarea laboral' en una dimensión diferente que nosotros calificaríamos de cognitiva y social.

Es esta nueva dimensión predominante en el sistema de producción la que comienza a superar cuantitativa, pero sobre todo cualitativamente, la noción de cualificación profesional, entendida como capacitación concreta y específica para el desempeño de tareas materiales bien definidas, prefijadas de antemano, de realización individual, rutinaria y mecánica (Buechtemann, 1999). En su lugar se comienza a hablar de competencia profesional y, sobre todo, personal, que combina la noción anterior en lo que se refiere al dominio de una serie de conocimientos teóricos y prácticos con un conjunto de exigencias, requisitos o cualidades más conectadas con la vertiente personal del trabajador, ya sea en sentido individual o colectivo, para responder así a los requerimientos de los entornos laborales, modos y procesos de producción de nuestro tiempo (CEDEFOP, 1994).

$Y$ en la medida en que este segundo complejo de conocimientos, actitudes y habilidades sociales, culturales y personales son aplicables a muchas y diversas situaciones ocupacionales y, en consecuencia, permiten la adaptación y flexibilidad del trabajador requerida hoy por la empresa y sus entornos, se comenzó a hablar también, con mayor o menor precisión terminológica, de competencias clave.

... it must be necessary and beneficial to any individual and to society as a whole. It must enable an individual to successfully integrate into a number of social networks while remaining independent and personally effective in familiar as well as new and unpredictable settings... a key competence must enable people to constantly update their knowledge and skills in order to keep abreast of fresh developments. (European Comission, 2002, p. 14)

De alguna forma se trata de un proceso de exteriorización de las interioridades del sujeto, con la consiguiente incorporación al sistema productivo de aquellos campos, espacios o funciones del sujeto que la ciencia de nuestro tiempo ha revalorizado y mostrado inevitables para el funcionamiento óptimo de las nuevas herramientas y procesos de producción. El "homo competens" sería en este sentido una reedición psicosocial del "homo economicus"; a esto quedaría reducido para algunos el interés que se viene demostrando por la dimensión cualitativa de los recursos humanos. Se trata de una visión crítica de este enfoque que es conveniente no perder de vista.

A estas alturas, se hacen inevitables algunas preguntas: ¿estamos en condiciones ahora, tras una década de aproximaciones 
empíricas, de trazar la estructura que conduce y soporta el nuevo mapa de exigencias formativas, sea cual fuere la denominación que reciban? ¿Cuál es su modelo y dinámica de generación? En nuestra opinión son tres las variables que se ponen en juego: el sujeto, el escenario laboral y el entorno, o, si se prefiere, dos: sujeto y entorno, cuya dinámica relacional se traslada al escenario laboral para su usufructo (García, 2002).

Alguien puede pensar que son las mismas variables del modelo anterior; no exactamente y mucho menos lo es su concepción, los componentes y dimensiones implicadas y, sobre todo, su comportamiento, la dinámica interrelacional que se postula entre ellas. El incremento y diversificación de esos componentes - materiales y personales, individuales y grupales, instrumentales, culturales y sociales -, dimensiones - cognitiva, procedimental, actitudinal, emocional - así como el carácter autopoyético de la dinámica relacional que se establece entre ellos, da como resultante un inventario amplísimo de competencias, algunas de las cuales parecen ajustarse a las exigencias de distintos escenarios laborales, puestos de trabajo y en distintos sectores. Ello redunda en beneficio de la empleabilidad de aquellos que las poseen y garantizan la necesaria adaptación a los cambios que se producen en el espacio y en el tiempo; por eso se las denomina competencias transversales, clave... en función del contexto sociocultural y laboral'5.

\section{Modos de adquisición y desarrollo de competencias}

Pasamos ahora a centrarnos en lo relativo a la forma como se adquieren y se desarrollan las competencias. Las respuestas que se proporcionen a estas preguntas constituyen buenos indicadores de si en realidad estamos ante una nueva forma de abordar las relaciones entre formación y trabajo o seguimos moviéndonos en esquemas anteriores.

¿Cómo, dónde y cuándo se adquieren, se desarrollan y transmiten esas capacidades? Para contestar a esta pregunta correctamente deberíamos de recorrer grupos o familias de competencias y grupos o familias de trabajos. No es nuestra intención entrar ahora en un análisis detallado de las estrategias susceptibles de ser utilizadas en cada una de ellas; para nuestro objetivo es suficiente con algunas consideraciones derivadas precisamente de lo que hemos llamado en el epígrafe anterior modelo de generación de competencias.

En primer lugar, queremos llamar la atención sobre la importancia que en este enfoque adquiere el llamado proceso de socialización primario, momento y proceso donde tiene lugar la configuración paulatina del conjunto de capacidades y actitudes generales que convergen en la formación de muchas de esas competencias, sobre todo de las llamadas competencias básicas y transversales. Es el primer indicador de que en esta perspectiva la escuela y la empresa, los escenarios formalizados ad hoc, estarían dejando de ser suficientes como instancias únicas y exclusivas de formación. Varias de esas capacidades, actitudes y habilidades, cognitivas, emocionales y sociales, también se adquieren vía relación sujetoentorno más o menos estructurada y, por supuesto, más allá de escenarios pensados específicamente con una intención formativa o laboral.

En segundo lugar, no debemos ignorar que en este enfoque el énfasis se pone en la acción más que en el pensamiento, en el uso de los conocimientos y habilidades más que en su tenencia, en el comportamiento del sujeto en situación más que en el conjunto de procesos abstractos y computacionales (Lave; Wenger, 1991); y en esa tesitura situacional se activan variables polimórficas y se recuperan conformaciones adquiridas en otros tiempos y espacios, en otras situaciones, incluidas aquellas que pueden no considerarse estrictamente académicas o laborales.

Y, en tercer lugar, una consideración estrechamente conectada con las anteriores. Algunas ca-

5. En la investigación a la que nos hemos referido se propuso una matriz competencial formada por varios campos o dominios, pertenecientes a cinco niveles diferentes, que daba como resultado un total de doce competencias transversales distribuidas entre los diversos campos. 
racterísticas de esas competencias, por ejemplo, el predominio del componente informacional en una sociedad que así se tipifica, puede generar la impresión de que el escenario o la forma más idónea de adquisición y fomento de esas capacidades y habilidades es el individual, cuando es precisamente lo contrario: son las formas sociales de enseñanza, aprendizaje y acción las que facilitan la adquisición y desarrollo de esas competencias en el individuo (Wenger, 2001). Este tipo de contenidos, si así puede hablarse, es más fácilmente asimilado por el sujeto cuando la tarea se realiza en grupo y, sobre todo, cuando la tarea realizada en grupo requiere de la colaboración y cooperación de todos los miembros del grupo; es como si en este nivel de competencias tomase concreción el principio pedagógico general de que el aprendizaje es un proceso que se desarrolla en el individuo pero que requiere la presencia de los demás; frente a los modelos de formación y de trabajo predominantemente individualistas y aislacionistas, el enfoque de las competencias requiere inevitablemente la consideración del otro, de lo otro y de los otros.

Pues bien, en base a estas tres reflexiones, que no acotan en absoluto la reflexión, entendemos que si las características económicas, técnicas y sociales de nuestro tiempo han llevado a pensar en la conveniencia de que el sujeto aporte unos nuevos contenidos en forma de competencias, unos contenidos que trascienden el componente específicamente técnico por haberse superado el reducido marco laboral, en sentido económico y conceptual, al que nos tenía acostumbrado el modo de producción industrial, la adquisición y transmisión de esos contenidos requiere a su vez de unos medios, métodos y hasta unas formas sociales diferentes de aprendizaje; esas formas y modos diferentes son los que están apuntando, y constituyen ya, un esquema distinto de pensar y fomentar la formación.

\section{La autoformación como modelo básico y competencia clave}

En la reflexión tradicional sobre educación y trabajo ha predominado un enfoque fragmenta- do en un doble sentido: separación de estos términos y su significado respecto de otros factores, facetas o dimensiones vitales del individuo y separación, a su vez, de esos términos entre sí para luego buscar puentes de conexión; esta forma de reflexión ha cristalizado en escenarios, ambientes, instancias educativas y laborales más bien estancas que, por más esfuerzos que se vienen haciendo en las últimas décadas para potenciar su permeabilidad, mantienen sus respectivas lógicas de funcionamiento y supervivencia.

La validez y continuidad de esta forma de reflexión radicaba en la estabilidad de los dos polos de la relación, incrementada a su vez por la propia reflexión y por los avances en los componentes esenciales de cada uno de los polos, el conocimiento y la tarea. Y, después de tanto tiempo de aplicación, nada más fácil que pensar que este modelo podía estirarse en el tiempo cuanto fuese necesario, con simples retoques y ajustes en uno $u$ otro sentido.

A este esquema respondía buena parte de la investigación que hasta hace poco se ha venido haciendo en nuestro campo. De ahí que una y otra vez encontremos la misma estructura del discurso, que suele comenzar por el análisis de las tendencias a las que está sometida la economía de nuestro tiempo para preguntarse a continuación por las reformas que de manera coherente deben introducirse en la educación.

Nosotros estamos proponiendo otra forma de reflexión, que va más allá de este enfoque lineal y que se apoya en la superación de la fragmentación del individuo y en su consideración como sujeto en situación. La investigación de esta segunda era moderna está empeñada en estudiar al sujeto en su totalidad, sea cual fuere el escenario en que se mueva. Dos aspectos - visión global del sujeto en situación - de suficiente trascendencia como para alterar sustancialmente la corriente hasta hace poco hegemónica.

No estamos, pues, ante meros cambios en el mundo económico que estén solicitando transformaciones en la formación de los futuros y actuales trabajadores; no se trata sólo de que nos estemos enfrentando a nuevos modelos 
organizacionales y formas productivas como consecuencia de la irrupción de nuevas tecnologías en los procesos de producción. Esta fase está hoy ya superada.

Es la propia sociedad, no sólo el trabajo o la educación, quien está sometida a nuevas formas de organización, estructuración, como resultado ciertamente de la aparición de nuevos modos y medios de comunicación e información que posibilitan la confluencia de factores y dimensiones hasta ahora separados en el tiempo y en el espacio, pero también como fruto de la necesidad de estudiar los fenómenos, sea cuales fueren, en su globalidad y carácter interrelacional. Podríamos decir que la destaylorización ha traspasado el marco de las relaciones productivas, y también el de las formativas, para afectar al conjunto de la dinámica social, de la vida. Las fronteras entre lo uno y lo otro - trabajo, formación y vida - ya no son impermeables, al contrario, predomina el solapamiento y cruce de campos y dimensiones. La unidad de análisis de los fenómenos socioeducativos ya no se decide en función de la actividad predominante en un momento dado de la vida del sujeto, sino en base a la situación que ocupa en un entorno concreto.

De ahí que los enfoques educativos en base al principio de la formación continua entendido en sentido lineal, educación a lo largo de la vida, hayan quedado periclitados. Frente a ello se empieza a hablar también de educación a lo ancho de la vida (Vázquez, 2002), pues la educación no sólo ha dejado de tener un tiempo sino también un espacio exclusivo. Más aún, la educación en esta sociedad habría dejado de corresponder a un tipo concreto de acción; éste es el concepto de sociedad educativa, de redes de formación, de comunidades de aprendizaje y, sobre todo, de comunidades de práctica.

Pues bien, si las tareas formativas y los cometidos laborales están conociendo en la sociedad de nuestros días un proceso de deslocalización y solapamiento, combinación y mezcla de funciones, tiempos y actividades, si además los perfiles de esas tareas y cometidos se han ampliado, diversificado en una línea más social y práctica, situacional la hemos llamado anteriormente, cabe preguntarnos si no procede empezar a hablar y pensar la formación bajo otro modelo que bien podríamos calificar de autoformación en el sentido de conceder protagonismo al sujeto para detectar sus necesidades formativas y valorar los modos, lugares y tiempos de tratarlas, en vez de seguir depositando esas decisiones en manos de unas instancias pensadas ad hoc por una sociedad rígidamente estructurada y compartimentalizada en todos los órdenes de la vida, también por supuesto en lo que se refiere a formación, trabajo y la relación entre estos dos términos.

Autoformación aquí no equivale a individualismo, independencia, aislamiento y desorden (Gauthier, 2001); tiene más bien el sentido de capacidad del sujeto para autogestionar su proyecto de vida en relación con su entorno, enfatizando las ideas de globalidad e interrelación que antes mencionábamos, pues es todo el sujeto quien se construye en cada situación.

Más concretamente, en el contexto que nos ocupa autoformación hace referencia a esa capacidad del sujeto para detectar, valorar y decidir qué perfil de formación necesita y dónde y cómo puede desarrollarlo. En este sentido la autoformación vendría a ser una de las competencias que en este trabajo hemos calificado de transversales, más aún, de la matriz competencial la autoformación sería la competencia clave, que a su vez estaría formada por un conglomerado de dimensiones, cognitivas y emocionales, individuales y grupales, personales, materiales y culturales, procedimentales y actitudinales, críticas y valorativas de las propias capacidades, habilidades, recursos y limitaciones.

El desarrollo de esta competencia en toda su complejidad nos llevaría a un segundo significado relativo ahora a una forma de gestionar los derroteros formativos de la sociedad en su conjunto, un modelo exigido no tanto por las características económicas de nuestro tiempo como por la estructura, trama y dinámica social. Una sociedad como la nuestra 
no requiere sólo de una adaptación permanente de contenidos y perfiles formativos y laborales propuestos por otros sino poner al sujeto, y a la propia sociedad, en condiciones para diagnosticar, valorar y orientar sus propias necesidades formativas como trabajador y ciudadano, constructor de la misma sociedad.

\section{Concluyendo}

Hemos identificado y descrito brevemente la autoformación en dos sentidos: como competencia clave y en cuanto modelo básico de formación en la sociedad de nuestro tiempo. Ahí hemos llegado dando un paso más allá del mero inventario de competencias, que empieza ya a abundar en la literatura científica; sobreponiéndonos a los simples listados de competencias, nos hemos preguntado por la forma como se generan, adquieren y desarrollan, concluyendo que el enfoque de la autoformación responde no sólo a determinadas tendencias económicas sino a las transfor- maciones que viene conociendo la sociedad en la última década.

Si detrás del enfoque economicista de la autoformación se encuentra una concepción tradicional de la educación, más o menos activa pero individualista, didactista, psicologicista y escolar, esta otra interpretación más amplia y social del proceso de autoformación implica, en cambio, una concepción y dinámica diferente, más interrelacional, menos funcionalista y escolar. Si apostamos por hablar y defender como principio educativo la formación a lo largo de toda la vida, la mejor forma de promoverlo es poniendo al sujeto en condiciones de formarse por sí mismo, de autoformarse, de manera que la autoformación pasaría a ser la llave maestra de uno de los pilares educativos de nuestro tiempo; utilizando terminología más actual, la autoformación vendría a ser la locomotora del convoy de competencias formativas exigibles hoy a cualquier individuo, con lo que se nos presenta desde el primer momento como la competencia clave de la formación en el siglo XX1.

\section{Referencias bibliográficas}

ALBERT, C. et al. Del sistema educativo al mercado de trabajo: un análisis de flujos. Revista de Educación, Madrid, v. 330, p. 137$155,2003$.

ALLES, M. A. Gestión por competencias: el diccionario. Buenos Aires: Granica, 2005.

Autoempleo: una salida para el desempleo. Buenos Aires: Granica, 2006.

BUECHTEMANN, C. El cambio en la demanda de cualificaciones. Revista Europea de Formación Profesional, Berlín, v. 16, p. 67 70, 1999

CARRÉ, P.; MOISAN, A. ; POISSON, D. L'autoformation. Paris: PUF, 1997.

CASAL, J.; COLOMÉ, F.; COMAS, M. La interrelación de los tres subsistemas de Formación Profesional en España. Madrid: Fundación tripartita para la Formación en el empleo/Fondo Social Europeo, 2003.

COMISIÓN EUROPEA. Crecimiento, competitividad y empleo: retos y pistas para entrar en el siglo XXI. Libro Blanco. Luxemburgo: Oficina de Publicaciones Oficiales de las Comunidades Europeas, 1993.

Afrontar Los cambios en el trabajo. Luxemburgo: Oficina de Publicaciones Oficiales de las Comunidades Europeas, 1999a.

Ayudar a los jóvenes en su transición de la escuela al trabajo. Luxemburgo: Oficina de Publicaciones Oficiales de las Comunidades Europeas, 1999b. 
. La estrategia europea del empleo - invertir en las personas: invertir en más y mejores trabajos. Luxemburgo: Oficina de Publicaciones Oficiales de las Comunidades Europeas, 1999c.

Un nuevo impulso para la juventud europea. Libro Blanco de la Comisión Europea. Luxemburgo: Oficina de Publicaciones Oficiales de las Comunidades europeas, 2002.

. Trabajar juntos a nivel local para crear más y mejores empleos para todos: la dimensión local de la estrategia europea para el empleo. Luxemburgo: Oficina de Publicaciones Oficiales de las Comunidades Europeas, 2003.

FERNÁNDEZ, G. Las competencias: clave para una gestión integrada de los recursos humanos. Bilbao: Deusto Ediciones, 2006.

CEDEFOP. Las competencias: el concepto y la realidad. Revista Europea de Formación Profesional, Berlín, v. 1. Monográfico, 1994.

EUROPEAN COMMISION. Key competencies. Brussels: Eurydice, 2002.

FUJII, G.; RUESGA, S. M. (Coords.). El trabajo en un mundo globalizado. Madrid: Pirámide, 2004.

GARCÍA, Á. Educación y ciudadanía. Reconstrucción del problema en términos de relación. En: FERNANDES MAIA, C. (Org.). Educação e Cidadania. Chaves: Sindicato Nacional dos Profesores Licenciados, 2002, p. 31-57.

GARCÍA, Á.; MARTÍN, A. V.; PÉREZ, M. D. Movilidad y competencias clave: exigencias de la formación en el siglo XXI. En: RODRÍGUEZ, T.; PEÑA, J. V.; HERNÁNDEZZ, J. (Orgs.). Cambio educativo: presente y futuro. Universidad de Oviedo: Servicio de Publicaciones, 2000, p. 177-198.

GAUTHIER, P. Didier: ingénierie pédagogique de l'autoformation éducative - l'etat de l'art de l'autoformation et des nouvelles technologies éducatives pour des entreprises cognitives. LE CADRE D'UN DESS INGENIERIE DE FORMATION DE L'UNIVERSITE DE ROUEN ET DU CNED, 2001. Disponible en : <http://membres.lycos.fr/autograf/INGENIERIE_AUTOFORMATION.htm>. Consultado en: 25 abr. 2006.

HAMMER, M.; CAHMPY, J. Reingeniería de la empresa. Barcelona: Paramon, 1994.

HOMS, 0.; RESINES, D. Educación, trabajo y sociedad del aprendizaje. En: TEDESCO, J. C. Por una ciudad comprometida con la educación. Barcelona, v. 1, 1999, p. 327-339.

IZQUIERDO, F. J. El teletrabajo. Madrid: Difusión jurídica y temas de actualidad, 2006.

JOHNES, G. Economía de la educación: capital humano, rendimiento y mercado de trabajo. Madrid: Ministerio de Trabajo y Seguridad Social, 1995.

LAVE, J.; WENGER, E. Situed Learning. Cambridge: University Press, 1991.

LÁZARO, L. M.; MARTíNEZ, M. J. Educación, empleo y formación en la Unión europea. Valencia: Universitat de Valencia, 1999.

LOPE, A.; MARTÍN, A. Las relaciones entre formación y empleo: ¿qué formación para qué empleo? Economía y Sociología del Trabajo, Madrid, v. 27/28, p. 254-266, 1995.

LOPEZ, M. Á.; GRANDIO, A. (Coords.). Capital humano como fuente de ventajas competitivas: algunas reflexiones y experiencias. La Coruña: Netbibro, 2005.

MARTIN, J.-P. ; SAVARY, É. Invertir en formación. Lyon: Cronique Sociale, 1998.

MORA, J. G. Competencias y empleo de los jóvenes titulados universitarios. Revista de Educación, Madrid, v. 330, p. 157-170, 2003.

MORENO, G. Trabajo y ciudadanía: un debate abierto. Vitoria-Gasteiz: Ararteko, 2003.

PETIT, A.; BADOSA, J.; MAMPEL, J. Educación y escuela: el nuevo papel de la escuela dentro de una concepción educativa más 
amplia. Los proyectos educativos de ciudad. En: SUBIRATS, J. (Coord.). Gobierno local y educación: la importancia del territorio y la comunidad en el papel de la escuela. Barcelona: Ariel, 2002, p. 199-223.

RODRÍGUEZ, J. La transformación de la sociedad salarial y la centralización del trabajo. Madrid: Talasa, 2006.

SARRAMONA, J. Las competencias básicas de la enseñanza obligatoria. Barcelona: CEAC, 2004. La formación continua laboral. Madrid: Biblioteca Nueva, 2002.

Competencias básicas al terminar la escolaridad obligatoria. Revista de Educación, Madrid, v. 322, p. 255-288, 2000.

TAGLIAGAMBE, S. Competencia y capacidades en la reforma del sistema escolar. Revista de Educación, Madrid, n. extraordinario, p. 83-101, 2002.

U.G.T. Boletín de empleo. Madrid: Secretaría Ejecutiva General, Departamento Confederal de la Juventud Trabajadora, 2004.

VÁZQUEZ, G. El sistema educativo ante la educación de calidad para todos a lo largo y ancho de la vida. Revista de Educación, Madrid, n. extraordinario, p. 39-57, 2002.

WENGER, E. Comunidades de práctica: aprendizaje, significado e identidad. Barcelona: Paidos, 2001.

WILSON, D. N. La reforma de la formación técnica y profesional para un mundo laboral cambiante. Perspectivas, Ginebra, v. XXXI/ 1, p. $21-40,2001$.

Recibido en 21.06.06

Aprobado en 05.03.07

Ángel García del Dujo es Licenciado y Doctor en Filosofía y Ciencias de la Educación por la Universidad de Salamanca. Profesor Titular de la Universidad de Salamanca. Autor de diversos libros y artículos en revistas científicas. Sus líneas de investigación: los procesos educativos, la educación no formal y la fundamentación pedagógica de las nuevas tecnologías.

José Manuel Muñoz Rodríguez es Licenciado y Doctor en Pedagogía por la Universidad de Salamanca. Premio Extraordinario de Doctorado. Profesor Ayudante de la Universidad de Salamanca. Sus líneas de interés se centran en la educación ambiental, la pedagogía laboral y la educación no formal, con aportaciones en revistas científicas. 